\title{
The Necessary Conditions Imposed on the Coefficients of Multistep Hybrid Methods Adapted for Solving ODEs of the Second Order
}

\author{
VUSALA NURIYEVA \\ Department of Computational Mathematics \\ Baku State University \\ Z.Khalilov 23 \\ AZERBAIJAN
}

\begin{abstract}
There are some classes of methods to solve the initial-value problem for the ODEs of the second order. Recently among of them are developed the numerical methods, which are using in the application of computer technology. By taking into account the wide application of the numerical methods, here has investigated the numerical solution of the above-mentioned problem. For this aim here has constructed the multistep hybrid method with the special structure, which has been applied to solve the initial-value problem of the ODEs of the second order. Given some recommendation to choosing of the suitable methods for solving above named problem and also, have found some bounders imposed on the coefficients of the convergence methods. Constructed specific methods solve the initial-value problem for ODEs of the second order. The received theoretical results have been illustrated by using some concrete methods, which have applied to solve model problem for ODEs of the second order.
\end{abstract}

Key-Words-ODEs of the Second Order, multistep method of the constant coefficient, convergence and degree, hybrid methods.

Received: February 28, 2021. Revised: June 22, 2021. Accepted: July 15, 2021. Published: August 10, 2021.

\section{Introduction.}

Many scientific and engineering problems are reduced to solving the initial-value problem for ODEs of the second order. To find the numerical solution of this problem are used in basically one-step and multistep methods. Among of them more known are Runge-Kutta and Adams-Bashford or Adams Moulton methods. All methods have their advantages and disadvantages. Because some scientists decide to construct methods on the intersection of the Runge-Kutta and Adams methods, which have call as the hybrid methods (see for example [1]-[16]). The specialists have proved that the stable methods of hybrid type are more exact than the others. This theory has been demonstrated by using some of Runge-Kutta and Adams methods. Have proved that the hybrid methods are more exact and in some cases, they have extended stability region. Note that these methods also have some disadvantages. For the demonstration of some properties of these methods, let us consider to the following problem:

$$
\begin{aligned}
& y^{\prime \prime}=f\left(x, y, y^{\prime}\right), y\left(x_{0}\right)=y_{0}, y^{\prime}\left(x_{0}\right)=y_{0}^{\prime}, \\
& x_{0} \leq x \leq X
\end{aligned}
$$

Suppose that this problem has the unique solution defined on the interval $\left[x_{0}, X\right]$. The aim of investigation dedicated in the construction of the stable numerical methods with the higher order of accuracy and application them to solve problem (1). Therefore the segment $\left[x_{0}, X\right]$ is divided to $N$ equal parts by using mesh points $x_{i+1}=x_{i}+h(i=0,1, \ldots, N)$, here $0<h$ is the step-size. And also suppose that the function $f(x, y, z)$ is continuous to totality of arguments, which has defined on the domain 
$D=\left\{x_{0} \leq x \leq X, \max (|y|,|z|) \leq A\right\} \quad$ and has the continuous partial derivatives up to $p$, inclusively (here $p$ is the degree of the investigated method). It is evident that by using variable replacement substitution the problem (1) can be reduced to the system of initial-value problem for ODEs of the first order. To solving of this system one can be used the multistep methods. One of the popular methods for solving initial-value problem for ODEs of the first order is the multistep methods with constant coefficients, which can be written as the following:

$$
\sum_{i=0}^{k} \alpha_{i}^{\prime} y_{n+i}=h \sum_{i=0}^{k} \beta_{i}^{\prime} y_{n+i}^{\prime} \quad(i=0,1, \ldots, N-k) \text {. }
$$

(2)

This method can be applied to solving of initial-value problem for the ODEs of the first order by using the equalities $y_{m}^{\prime}=f\left(x_{m}, y_{m}\right) \quad(m=0,1,2, \ldots)$. Method has fundamentally investigated by some authors (see for example [1]-[18]). By using the generalization of the method (2) one can be constructed multistep method in the following form (see for the example [7]-[13]):

$$
\begin{aligned}
& \sum_{i=0}^{k} \alpha_{i} y_{n+i}=h \sum_{i=0}^{k} \beta_{i} y_{n+i}^{\prime}+h^{2} \sum_{i=0}^{k} \gamma_{i} f_{n+i} \\
& \left(f_{m}=f\left(x_{m}, y_{m}, y_{m}^{\prime}\right), m \geq 0\right) .
\end{aligned}
$$

(3)

It is clear that for the application of the method

(3) to solve the problem (1), the values of $y_{m}$ and $y_{m}^{\prime},(m=0,1, \ldots, N)$ must be known. To find these values one can use the method of (2). Thus receive that by using method (2) and (3) one can solved the problem (1). As is known for comparison of numerical methods are used in basically the conceptions of stability and the degree. By taking into account this, let us define the mentioned conceptions.

Definition1: The method (2) is called as the stable, if the roots of the polynomial $\hat{\rho}(\lambda)=\alpha_{k}^{\prime} \lambda^{k}+\alpha_{k-1}^{\prime} \lambda^{k-1}+\ldots+\alpha_{1}^{\prime} \lambda+\alpha_{0}^{\prime}$ are located in the unit circle on the boundary of which there are no multiply roots.

If take into account that the linear part for these methods are written as the one and the same form, but they can be receive the different values (in means, that, these coefficients are fined by the one and the same way, therefore them can get one and the same values), then receive that the conception of "stability" for these methods (2) and (3) can be defined by the same way. Because, let us consider to determination of the conception of degree:

Definition 2: Integer value $p$ is called as order of the method (3), if the following is hold:

$\sum_{i=0}^{k}\left(\alpha_{i} y(x+i h)-h \beta_{i} y^{\prime}(x+i h)-h^{2} \gamma_{i} y^{\prime \prime}(x+i h)\right)=$ $=O\left(h^{p+1}\right), h \rightarrow 0$.

(4)

If in the asymptotic equality (4) put the $\gamma_{i}=0(i=0,1, \ldots, k)$, then from that receive the definition of the conception degree for the method (2).

Note that if the method (2) is stable then there are methods of the type (2) with the degree $p \leq 2[k / 2]+2$, but if the method (3) is stable, then there are methods with the degree $p \leq 2 k+2$ (see for example [8]-[11], [17], [18]). Application of the methods (3) to solving initial-value problem (1) by using the method (2) remind the using predictorcorrector method. It is known that if corrector method has the degree $p$, then degree for the predictor method must be not less than $p-1$. From here receive that for the maintaining accuracy of the method of type (3), which have used the method (2) for calculation of the values $y_{m}^{\prime}(m \geq 0)$ must be unstable, for the having the degree $p>k+2$. Noted that in the class of methods of the type (2) there are methods with the degree $p \leq 2 k$. But methods with degree $p>k+2$ are unstable. From here receive that by using the methods (2) and (3) one can be construct methods for solving problem (1) with the degree $p \leq 2 k+1$. It is known that some authors proposed to use the stable methods of type (2). In this case the rate of approaches for constructed methods by using methods (2) and (3) will be satisfies the condition $p \leq k+3$.

\section{Construction more exact stable methods with constant coefficients.}


It is known that the maximal degree for the stable methods of the type (2) equal to $P_{\max }=k+2$. Therefore some specialists have proposed to change the method (2) by the hybrid methods, taking into account that the hybrid methods are more exact. We will illustrate this case for the initial-value problem of ODEs of the second order with special structure. It is known that some of problems of the natural sciences are reduced to solving of the problem:

$$
\begin{aligned}
& y^{\prime \prime}=g(x, y), y\left(x_{0}\right)=y_{0}, y^{\prime}\left(x_{0}\right)=y_{0}^{\prime}, \\
& x_{0} \leq x \leq X .
\end{aligned}
$$

(5)

This problem in scientific literature has called as the initial-value problem for the ODEs of the second order with special structure. One of the effective methods for solving of considered problem (5) is the Stermer method, which can be written as (see for example [1], [7]-[10]):

$$
\begin{aligned}
& \sum_{i=0}^{k} \alpha_{i} y_{n+i}=h^{2} \sum_{i=0}^{k} \gamma_{i} g_{n+i}\left(g_{n}=g\left(x_{n}, y_{n}\right) ;\right. \\
& n=0,1, \ldots, N-K) .
\end{aligned}
$$

It is known, that method (6) fundamentally has investigated for the values of the coefficients which can be presented with as the following:

$$
\begin{array}{ll}
\alpha_{k}=1, \alpha_{k-1}=-2, & \alpha_{k+2}=1, \\
\alpha_{k-3}=\ldots=\alpha_{0}=0 &
\end{array}
$$

Noted, that in the case $\alpha_{k} \neq 0 \quad \gamma_{k} \neq 0$

It follows from (6) that in this case will be implicit. As it is known, in this case the application of the method (6) arises some difficulty. For solving this problem, one can be used predictor-corrector method. Because here is not investigated the convergence of the predictor-corrector method. Taken into account that this question investigated in the [20]. Note, that the method (6) also can be received from the method (3) as the partial case. As was noted, method (3) has applied to solving many practical problems, so as in this class methods one can be find the stable methods with the degree $\mathrm{p}=2 \mathrm{k}+2$. Method 3 in more general form investigated in the work [8]. In solving some problems arises in the necessary in the construction table methods with the degree $p>2 k+2$. For the same here was investigated the hybrid methods which applied for solving problems (5). It is known, that all the methods have its advantages and disadvantages. Therefore these methods are the independent objects for the investigation. For the construction of more exact methods, let us to write the method (6) in the following form:

$$
\sum_{i=0}^{k} \alpha_{i} y_{n+i}=h^{2} \sum_{i=0}^{k} \gamma_{i} g_{n+i+v_{i}}\left(\left|v_{i}\right|<1, i=0,1, \ldots, k\right) .
$$

(7)

This method is call as the hybrid and different from the others that, in these methods has used the values $y_{n+i+v_{i}},\left|v_{i}\right|<1, i=0,1, \ldots, k$. And the conception of degree for this method can be defined by the following definition.

Definition 3: The integer value $p$ calls as the degree of the method (7), if the following is hold:

$$
\begin{aligned}
& \sum_{i=0}^{k}\left(\alpha_{i} y(x+i h)-h^{2} \gamma_{i} y^{\prime \prime}\left(x+\left(i+v_{i}\right) h\right)\right)= \\
& O\left(h^{p+2}\right), h \rightarrow 0 .
\end{aligned}
$$

Here $x=x_{0}+n h$-fixed points.

For the determining some properties of the coefficients of the method (7) let us consider to following section.

\section{The necessary conditions of the convergence for method (7).}

As is known, method (6) investigated fundamentally by Dahlquist (see [18]). For the objectivity let us noted, that the maximal value for the degree of the method (6) for the stable and unstable methods had define by Dahlquist (see [18]). One of the basic questions is the determined the necessary condition for it is convergence. For the method (6) this question solved by Dahlquist. But here we want to receive similarly results for the method (7) have received there. In first let us define the necessary condition imposed on the coefficients of the method (7). For this suppose that the method (7) is convergent. Prove that in this case its coefficients will satisfy to the following conditions:

A. The coefficients $\alpha_{i}, \gamma_{i}, v_{i}(i=0,1, \ldots, k)$ are real numbers and $\alpha_{k} \neq 0$.

B. The characteristic polynomials $\rho(\lambda) \equiv \sum_{i=0}^{k} \alpha_{i} \lambda^{i} ; \sigma(\lambda) \equiv \sum_{i=0}^{k} \gamma_{i} \lambda^{i+v_{i}}$ have no common factor different from constant. 
C. The conditions $\sigma(1) \neq 0$ and $\rho \geq 1$ are hold.

Noted, that the proving of three these conditions $\mathrm{A}, \mathrm{B}$ and $\mathrm{C}$ differs from the proving, which have used by Dahlquist. For example, in the proving of the convergence method (7) here has used the theory of final difference equation with the constant equation. See for example (21), (22) and etc. The needed for these conditions for the method (3) has been proved by Dahlquist (see [17]-[19]).

If take into account that the values of the solution of our problems are real numbers, then receive that the condition A must be satisfied. But the condition $\alpha_{k} \neq 0$ related with the defined value $y_{n+k}$ from the equality (7). Now let us to consider the condition $\mathrm{B}$ and suppose that the polynomials $\rho(\lambda)$ and $\sigma(\lambda)$ has the common factor, which has denoted as the polynomial $\varphi(\lambda) \neq$ const . It is evident that the equality of (7) by using the shift operator $E y(x)=y(x+h)$ or $\left.E^{v} y(x)=y(x+v h)\right)$ can be written as following:

$$
\rho(E) y_{n}=h^{2} \sigma(E) y_{n}^{\prime \prime} \text {. }
$$

The equality (9) is the finite-difference equation of the order $k$. It is known that, if the $k$-initial values known, then the finitedifference equation of (9) has unique solution. By taking into account assumption, receive that the equality (9) can be written as:

$$
\varphi(E)\left(\rho(E) y_{n}-h^{2} \sigma(E) y_{n}^{\prime \prime}\right)=0 \text {. }
$$

From here receive:

$$
\rho_{1}(E) y_{n}-h^{2} \sigma_{1}(E) y_{n}^{\prime \prime}=0 \text {, }
$$

here $\rho(\lambda)=\varphi(\lambda) \rho_{1}(\lambda), \sigma(\lambda)=\varphi(\lambda) \sigma_{1}(\lambda)$,

If the equation of $(10)$ has the unique solution, then receive that the finite-difference equation (9) will be have unique solution.

The finite-difference equation of (10) has the order less than $k$, but the finite-difference methods (9) and (10) are equivalent. Therefore the equation of (9) has the unique solution for the known initial-value, the quantity for which is less than $k$. From the theory of finitedifference methods, it is known that if known $k_{1}$ - initial values, then the finite difference equation with the order of $k_{1}$ will be have the unique solution. From here receive, that the finite difference equation of the order $\mathrm{k}(\mathrm{k}>$ $k_{1}$ ) has the unique solution for the initialvalue amount for which less than $\mathrm{k}$. From here receive that our assumption is not correct. Therefore the condition B is hold.

Now let us consider to the condition $\mathrm{C}$.

If in the equality of (9) to pass limit for approaches step-size $h \rightarrow 0$, then receive:

$\rho(1) y(x)=0$,

Here $x=x_{0}+n h$ is fixed point. It is not difficult to understand that from here it follows:

$\rho(1)=0$.

(11)

This is called as the necessary condition for the convergence of the method (7). By taking into account these conditions, let us the equality of (9) to write in the following form:

$\rho_{1}(E)(E-1) y_{n}-h^{2} \sigma(E) y_{n}^{\prime \prime}=0$.

By some simplification here, receive the following:

$\rho_{1}(E)\left(y_{i+1}-y_{i}\right) h^{-1}-h \sigma(E) y_{i}^{\prime \prime}=0$.

From the equality of (12) receive:

$\rho_{1}(E) y_{i}^{\prime}-h \sigma(E) y_{i}^{\prime \prime}=0$,

(13)

Here

$\rho_{1}(\lambda)=\frac{\rho(\lambda)-\rho(1)}{\lambda-1}, \quad \lim _{\lambda \rightarrow 1} \rho_{1}(\lambda)=\rho^{\prime}(1)$.

By using this in the equality of (13) receive: $\rho^{\prime}(E) z_{i}-h \sigma(E) z_{i}^{\prime}=0 \quad\left(z(x)=y^{\prime}(x)\right)$.

If in the equality of (14) pass to limit for $h \rightarrow 0$, then receive that $\rho^{\prime}(1) z(x)=0$ ( $x$ is fixed point). From this equality it follows that $\rho^{\prime}(1)=0$.

(15)

By this way prove that if the method (7) is convergent then $\rho(1)=\rho^{\prime}(1)=0$ it is to say that $\lambda=1$ is twice rooting for the polynomial $\rho(\lambda)$. Therefore the equality (14) can be written as:

$\rho_{1}^{\prime}(E)\left(z_{i+1}-z_{i}\right)-h \sigma(E) z_{i}^{\prime}=0$.

(16)

If in the equality (14) change variable $i$ from 0 (zero) to $n$ and by summing of the receiving equalities we have: 
$\rho_{1}^{\prime}(E)\left(z_{n+1}-z_{0}\right)=h \sigma(E) \sum_{i=0}^{n} z_{i}^{\prime}$.

If in the equality of (17) pass to limit for the $h \rightarrow 0$, then receive:

$\rho_{1}^{\prime}(1)\left(z(x)-z\left(x_{0}\right)\right)=\sigma(1) \int_{x_{0}}^{x} z^{\prime}(s) d s$,

Here $\mathrm{x}=x_{0}+n h-$ fixed point.

This equality can be written as:

$$
\begin{aligned}
& \rho^{\prime \prime}(1)\left(z(x)-z\left(x_{0}\right)\right)=\sigma(1) \int_{x_{0}}^{x} f(s, y(s)) d s \\
& \left(z^{\prime}(x)=y^{\prime \prime}(x) ; y^{\prime \prime}(x)=f(x, y)\right) .
\end{aligned}
$$

By our assumption receive that:

$$
\rho^{\prime \prime}(1)=\sigma(1) \text {. }
$$

(18)

It follows from here, that the necessary condition, written by the equalities of (11) and (15)for convergence of the method (7), can be written as the following form:

$$
\rho(1)=\rho^{\prime}(1)=0 \text {. }
$$

From this relation receive, that $\lambda=1$ is twice rooting for the polynomial $\rho(\lambda)$. One can prove that from the condition (19) it follows that the following is hold:

$$
\sum_{i=0}^{k}\left(\alpha_{i} y(x+i h)-h^{2} \gamma_{i} y^{\prime \prime}\left(x+\left(i+v_{i}\right) h\right)\right)=O\left(h^{p+2}\right),
$$$$
h \rightarrow 0
$$

$(p \geq 0)$

If this asymptotic equality is hold, then integer $p$ is called as the degree for the method (7).

Let us note that by using the condition (19) and (20) receive that $p \geq 1$. If $\sigma(1)=0$, then from the (18) receive: $\rho^{\prime \prime}(1)=0$. And now prove that if $\lambda=1$ is twice root of the polynomial $\rho(\lambda)$, then the method (7) doesn't convergent.

Let us method (7) write as the finite-difference equations by using the equality (14). In this case, receive:

$$
\begin{aligned}
& \bar{\alpha}_{k} z_{n+k-1}+\bar{\alpha}_{k-1} z_{n+k-2}+\ldots+\bar{\alpha}_{1} z_{n+1}+\bar{\alpha}_{0} z_{n}= \\
= & h \psi\left(x_{n+i+v_{i}}, z_{i_{n+i+v_{i}}}^{\prime}\right) .
\end{aligned}
$$

It is known that the general solution of nonhomogeneous finite-difference equation can be written as following:

$$
z_{m}=\bar{z}_{m}+z_{m}^{*}
$$

$\bar{z}_{m}$-general solution of homogeneous and $z_{m}^{*}$ is a partial solution of nonhomogeneous equations. It is not difficult to prove that the following is hold:

$\lim _{h \rightarrow 0} z_{m}^{*}=0$.

Therefore the behavior of the solution $z_{m}$ depends on the behavior of the solution $\bar{z}_{m}$. It is known that if all roots of polynomial $\bar{\rho}(\lambda) \equiv \alpha_{k-1} \lambda^{k-1}+\alpha_{k-2} \lambda^{k-2}+\ldots+\alpha_{1} \lambda+\alpha_{0}$ are real and different from each other's, then its solution $\bar{z}_{m}$ can be presented as:

$$
\bar{z}_{m}=C_{1} \lambda_{1}^{m}+C_{2} \lambda_{2}^{m}+\ldots+C_{k-1} \lambda_{k-1}^{m} \text {. }
$$

The coefficients $C_{i}(\mathrm{i}=1,2, \ldots, \mathrm{k}-1)$ can be determined by using the initial values, the amount of them is equal to $\mathrm{k}-1$.

If a lot of roots are multiply, then presentation (21) can be written as follows:

$$
\begin{aligned}
& \bar{z}_{m}=C_{1} \lambda_{1}^{m}+C_{2} \lambda_{2}^{m}+\ldots+C_{j} \lambda_{j}^{m}+C_{j+1} m \lambda_{j}^{m}+ \\
& +C_{j+2} m^{2} \lambda_{j}^{m}+C_{j+3} \lambda_{j}^{m} \ldots+C_{k-1} \lambda_{k-1}^{m},
\end{aligned}
$$

here suppose that the root $\lambda_{j}$ multiplied three times. From here receive that if $\lambda_{j}$ is double multiplying root, then the following is hold:

$\lim _{h \rightarrow 0} \bar{z}_{m}=\infty$.

(22)

By above described way prove that if the polynomial $\rho(\lambda)$ has the $\operatorname{root} \lambda=1$, which multiplied three times, then receive that $\lambda=1$ will be twice root for the polynomial $\bar{\rho}(\lambda)$. From here receive that if the polynomial $\rho(\lambda)$ has triple $\operatorname{root} \lambda=1$, then will hold correlation (22). It follows from here that the method will unstable. If $\rho(1)=\rho^{\prime}(1)=\sigma(1)=0$ then receive that $\lambda=1$ is triple root for the polynomial $\rho(\lambda)$ and therefore the method (5) is not convergent, which is contrary to our assumption. Thus prove that the condition $\mathrm{C}$ is hold.

\section{On the construction some of concrete methods and application one of them to solve model problem.}

It is not difficult to prove that if the method (7) has the degree $p$ then its coefficients must to 
satisfy the following conditions (see for example [3], [4], [7], [9]):

$$
\sum_{i=0}^{k} \alpha_{i}=0 ; \sum_{i=0}^{k} i \alpha_{i}=0 ; \sum_{i=0}^{k} \gamma_{i}=\sum_{i=0}^{k} \frac{i^{2}}{2 !} \alpha_{i}, \ldots
$$

$\sum_{i=0}^{k} \frac{l_{i}^{(p-1)}}{(p-1) !} \gamma_{i}=\sum_{i=0}^{k} \frac{i^{p+1}}{(p+1) !} \alpha_{i}, \quad l_{i}=i+v_{i}$ $(i=0,1, \ldots, k) \quad(23)$

Has proved that if coefficients of the method (7) satisfy to this system of nonlinear algebraic equations, then receive that the method (7) will be have the degree of $p$. Note that in the system (23) the quantity of unknowns equal to $3 k+3$, but the quantity of equations equal to $p+1$. This system is nonlinear because to find the exact solution of this system is not simple. Here have proved that there exist the stable methods of the type (23) with the degree $2 k+2$. The nonlinear system (23) is different from the known. It is known that the order of the method (7) must to satisfy the condition $p \leq 3 k+1$. If requested from the method (7) stability, then can prove that there are stable methods of type (7) with the degree $p=2 k+2$. Constructed have concrete method with the degree $p=6$ for $k=2$. In this case polynomial $\rho(\lambda)$ can be written as: $\rho(\lambda)=\lambda^{2}-2 \lambda+1$ in so far as $\lambda=1$ is twice rooting for the characteristic polynomial of the method (7). Let us in first constructed stable method of type (6) and after them to construct stable method of type (7) for the case $\mathrm{k}=2$. By taking into account the values $\alpha_{2}=$ $1 ; \alpha_{1}=-2 ; \alpha_{0}=1$ in nonlinear system (23), receive that the method with the maximal degree can be written as:

$$
y_{n+2}=2 y_{n+1}-y_{n}+h^{2}\left(g_{n+2}+10 g_{n+1}+g_{n}\right) / 12
$$

This method has received from the method of (6) in the case $k=2$. Noted that in this case maximal value for the degree of the method of (6) can be written as: $p \leq 2 k$. But if the method (6) is stable, then receive that there are methods with the degree $p \leq 2[k / 2]+2$. Thus receive that if $\mathrm{k}$-is even then there are the stable methods with the degree $p=k+2$ for all the $\mathrm{k}$. If consider the case $\mathrm{k}=3$, then by the Dahlquist results receive, that in this case
$P_{\max }=4$ for the stable methods. This method is one and the same with the method (24). And there is no stable methods with the maximal degrees and different from method (24), which is unique in this case $(\mathrm{k}=3)$. And now let us compose methods (2) and (6). Noted that the conceptions the stability and degree for these methods (stable and unstable) are one and the same. As is known impilict methods are as both scientific and practical interest. This result can be received from the following description. It is known, that if method (6) is stable and $\beta_{k}=0$, then $p \leq k$ (here p-degree, but $\mathrm{k}$ is the order). From here receive that stable implicit methods are more exactnees, than the explicit. But there arises some difficults in the application of the implicit methods to solving some problems. For solving this problem here proposed to use the predictor and corrector methods. We also will be used the predictor and corrector formulas for the aplication of the methods proposed here. For the construction predictor-corector methods have used the way, descripte in [1].

As was noted above for the construction more exactnees stable multistep method one can be used the method of (3) or (7). Taking into account, that in using of the methods of type (3) are arises some difficults, relayted with the calculation of the values $y_{m}^{\prime \prime}(m=0,1,2,3, \ldots)$. For this let us consider to construction methods of type (7) and put $\mathrm{k}=2$. By using the solution of the system (23), one can be constructed the following method:

$$
\begin{aligned}
& \quad y_{n+2}=2 y_{n+1}-y_{n}+h^{2}\left(5 f_{n+\gamma_{0}}+14 f_{n+\gamma_{1}}+\right. \\
& \left.+5 f_{n+\gamma_{2}}\right) / 24
\end{aligned}
$$

here $\gamma_{0}=1-\sqrt{10} / 5 ; \gamma_{1}=1 ; \gamma_{0}=1+\sqrt{10} / 5$. This method is unique, stable and has the degree $p=6$. Usually arise the question, which is reated with the calculation of the values having the type $y_{n+\gamma}(|\gamma|<1)$. Some authors offer to use hybrid method with the degree less than maximal. If in our case to use this recommendation, then one can be constructed the following method:

$$
y_{n+2}=y_{n+1}-2 y_{n}+h^{2}\left(4 f_{n+\gamma_{0}}+f_{n+\gamma_{1}}+4 f_{n+\gamma_{2}}\right) / 9 \text {, }
$$

here $\gamma_{0}=1-\sqrt{3} / 4 ; \gamma_{1}=1 ; \gamma_{0}=1+\sqrt{3} / 4$; 
In some cases for calculation of the value $y_{n+\gamma}$ can been used the known methods.

This method is stable and has the degree $p=4$ which was applied to solving of the following initial-value problem:

$y^{\prime \prime}(x)=\lambda^{2} y(x), y(0)=1, y^{\prime}(0)=\lambda, 0 \leq x \leq 1$,

exact solution of which define as: $y(x)=\exp (\lambda x)$.

The main goal is consist of compares constructed methods on the model problem for the simple case.

Table 1. Results receiving for the positive $\lambda$ and $h=0.1$ :

\begin{tabular}{|l|l|l|l|l|}
\hline$h=0.1$ & \multicolumn{2}{l|}{ Stormer } & \multicolumn{2}{l|}{ Method 26 } \\
\hline$x_{i}$ & $\lambda=1$ & $\lambda=5$ & $\lambda=1$ & $\lambda=5$ \\
\hline 0.2 & $\begin{array}{l}3.07 \mathrm{E}- \\
9\end{array}$ & $\begin{array}{l}7.18 \mathrm{E}- \\
5\end{array}$ & $\begin{array}{l}3.07 \mathrm{E}- \\
9\end{array}$ & $\begin{array}{l}7.18 \mathrm{E}- \\
5\end{array}$ \\
\hline 0.5 & $\begin{array}{l}3.45 \mathrm{E}- \\
8\end{array}$ & $\begin{array}{l}1.74 \mathrm{E}- \\
3\end{array}$ & $\begin{array}{l}3.45 \mathrm{E}- \\
8\end{array}$ & $\begin{array}{l}1.74 \mathrm{E}- \\
3\end{array}$ \\
& $\begin{array}{l}1.97 \mathrm{E}- \\
7\end{array}$ & $\begin{array}{l}5.22 \mathrm{E}- \\
2\end{array}$ & $\begin{array}{l}1.97 \mathrm{E}- \\
7\end{array}$ & $\begin{array}{l}5.22 \mathrm{E}- \\
2\end{array}$ \\
\hline 1.0 & & & & \\
\hline
\end{tabular}

In this table we have used the values of the function, which are increasing by the increasing of the variable $\mathrm{x}$. Noted that, the Stormer method and (26) has the same degree, but one of them has the hybrid type. Let us noted, that for the calculation of the values $y_{n+\gamma}$ we have used methods, which have the degree less than 3. But for the values calculated by Stormer method has been used method with the degree less than 4. No receiving results can be taking the same.

Table 2. Results receiving for the negative $\lambda$ and $\mathrm{h}=0.1$

\begin{tabular}{|l|l|l|l|l|}
\hline$h=0.1$ & \multicolumn{2}{l|}{ Stormer } & \multicolumn{2}{l|}{ Method 26 } \\
\hline$x_{i}$ & $\lambda=-1$ & $\lambda=-5$ & $\lambda=-1$ & $\lambda=-5$ \\
\hline 0.2 & $\begin{array}{l}2.51 \mathrm{E}- \\
9\end{array}$ & $\begin{array}{l}2.64 \mathrm{E}- \\
5\end{array}$ & $\begin{array}{l}2.51 \mathrm{E}- \\
9\end{array}$ & $\begin{array}{l}2.64 \mathrm{E}- \\
5\end{array}$ \\
& & & & \\
\hline
\end{tabular}

\begin{tabular}{|l|l|l|l|l|}
\hline 0.5 & $\begin{array}{l}2.32 \mathrm{E}- \\
8\end{array}$ & $\begin{array}{l}2.77 \mathrm{E}- \\
4\end{array}$ & $\begin{array}{l}2.32 \mathrm{E}- \\
8\end{array}$ & $\begin{array}{l}2.77 \mathrm{E}- \\
4\end{array}$ \\
\hline 1.0 & $\begin{array}{l}9.62 \mathrm{E}- \\
8\end{array}$ & $\begin{array}{l}3.60 \mathrm{E}- \\
3\end{array}$ & $\begin{array}{l}9.62 \mathrm{E}- \\
8\end{array}$ & $\begin{array}{l}3.60 \mathrm{E}- \\
3\end{array}$ \\
& & & & \\
\hline
\end{tabular}

Similarly results received for the case $\lambda<0$. It follows from here, that these methods are stable.

For the illustration the dependents of the errors from the step-size $0<\mathrm{h}$, in the following tables have investigated the numerical solution of the exercise (27) for the step size $\mathrm{h}=0.01$. In the results of which receive the same results. Noted, that if consider the case $\lambda=+-15$, than the method (26) can be give the best results.

Table 3. Results receiving for the positive $\lambda$ and $\mathrm{h}=0.01$

\begin{tabular}{|l|l|l|l|l|}
\hline$h=0.01$ & \multicolumn{2}{|l|}{ Stormer } & \multicolumn{2}{l|}{ Method 26 } \\
\hline$x_{i}$ & $\lambda=1$ & $\lambda=5$ & $\lambda=1$ & $\lambda=5$ \\
\hline 0.02 & $2.88 \mathrm{E}-$ & $4.56 \mathrm{E}-$ & $2.9 \mathrm{E}-$ & $4.56 \mathrm{E}-$ \\
& 15 & 11 & 15 & 11 \\
\hline 0.25 & $9.11 \mathrm{E}-$ & $2.32 \mathrm{E}-$ & $9.117 \mathrm{E}$ & $2.32 \mathrm{E}-$ \\
& 13 & 8 & -13 & 8 \\
\hline 0.5 & $4.12 \mathrm{E}-$ & $2.09 \mathrm{E}-$ & $4.12 \mathrm{E}-$ & $2.09 \mathrm{E}-$ \\
& 12 & 7 & 12 & 7 \\
\hline 0.75 & $1.04 \mathrm{E}-$ & $1.18 \mathrm{E}-$ & $1.04 \mathrm{E}-$ & $1.18 \mathrm{E}-$ \\
& 11 & 6 & 11 & 6 \\
\hline 1.0 & $2.12 \mathrm{E}-$ & $5.76 \mathrm{E}-$ & $2.12 \mathrm{E}-$ & $5.76 \mathrm{E}-$ \\
& 11 & 6 & 11 & 6 \\
& & & & \\
\hline
\end{tabular}

Table 4. Results receiving for the negative $\lambda$ and $\mathrm{h}=0.01$

\begin{tabular}{|l|l|l|l|l|}
\hline$h=0.01$ & \multicolumn{2}{l|}{ Stormer } & \multicolumn{2}{l|}{ Method 26 } \\
\hline$x_{i}$ & $\lambda=-1$ & $\lambda=-5$ & $\lambda=-1$ & $\lambda=-5$ \\
\hline 0.02 & $\begin{array}{l}2.55 \mathrm{E}- \\
15\end{array}$ & $\begin{array}{l}4.12 \mathrm{E}- \\
11\end{array}$ & $\begin{array}{l}2.60 \mathrm{E}- \\
15\end{array}$ & $\begin{array}{l}4.12 \mathrm{E}- \\
11\end{array}$ \\
\hline 0.25 & $\begin{array}{l}7.67 \mathrm{E}- \\
13\end{array}$ & $\begin{array}{l}1.01 \mathrm{E}- \\
08\end{array}$ & $\begin{array}{l}7.67 \mathrm{E}- \\
13\end{array}$ & $\begin{array}{l}1.01 \mathrm{E}- \\
08\end{array}$ \\
\hline 0.50 & $\begin{array}{l}2.95 \mathrm{E}- \\
12\end{array}$ & $\begin{array}{l}4.81 \mathrm{E}- \\
08\end{array}$ & $\begin{array}{l}2.95 \mathrm{E}- \\
12\end{array}$ & $\begin{array}{l}4.81 \mathrm{E}- \\
08\end{array}$ \\
& & & & \\
\hline
\end{tabular}




\begin{tabular}{|l|l|l|l|l|}
\hline 0.75 & $\begin{array}{l}6.38 \mathrm{E}- \\
12\end{array}$ & $\begin{array}{l}1.74 \mathrm{E}- \\
07\end{array}$ & $6.38 \mathrm{E}-$ & $1.74 \mathrm{E}-$ \\
& & & 07 \\
\hline 1.0 & $1.10 \mathrm{E}-$ & $6.11 \mathrm{E}-$ & $1.10 \mathrm{E}-$ & $6.11 \mathrm{E}-$ \\
& 11 & 07 & 11 & 07 \\
\hline
\end{tabular}

The receiving numerical results correspond to the theoretical results. For the simple comparison receiving results by using differential methods can be taken, that they are approximately the same.

\section{Conclusion.}

Here have constructed the hybrid methods of multistep type which are applied to solving of the initial-value problem for the ODEs of the second order with the special structure. To investigate this problem has been used a new way by which have been defined the necessary and sufficient conditions for the convergence of the constructed hybrid methods. By using the above-mentioned way we have compared the conception of stability using in the investigation of the methods (7) and (3). And also have constructed a formula similar to Dahlquist formula by which one can define the maximal value for the degree of the stable methods of the type (7). The way described here is new and therefore will find its followers.

\section{ACNOWLEDGMENTS}

The author express thank to the academicians Telman Aliyev and Ali Abbasov for their suggestion to investigate to the computational aspects of our problem.

\section{REFERENCES:}

[1] Vagif Ibrahimov, Mehriban Imanova, Galina Mehdiyeva, One relationship between Volterra integro-differential and ordinary-differential equations, AIP Conference Proceedings 2116, 2019

[2] Quarteroni, A., Sacco, R., Saleri, F. 2007. Numerical Mathematics, Springer. 2nd edition, p. 680

[3] Galina Mehdiyeva, Mehriban Imanova, Vagif Ibrahimov, An application of the hybrid methods to the numerical solution of ordinary differential equations of second order,
Vestnik KazNU, ser., math, mech., inf., 2012, No 4 (75). pp. 46-54

[4] Galina Mehdiyeva, Mehriban Imanova, Vagif Ibrahimov, On one advantage of hybrid methods, Kiev National University of Taras Shevchenko Journal of the Number of Applied MATHEMATICS, 2012.

[5] Pramod Pandey-A finite difference method for solution of nonlinear two point boundary value problem with Neumann boundary conditions. Transylvanian Journal of Mathematics and Mechanics

[6] E. O. Adeyefa, An Improved SelfStarting Implicit Hybrid Method, IOSR Journal of Mathematics, Volume 10, Issue 2 Ver.II (Apr.2014)

[7] Galina Mehdiyeva, Mehriban Imanova, Vagif Ibrahimov, A way to construct an algorithm using hybrid methods. Applied Mathematical Sciences, HIKARI Ltd,Vol. 7, 2013, no. 98 , pp.4875-4890.

[8] Vagif Ibrahimov, On a relation between order and degree for stable forward jumping formula. Zh. Vychis. Mat., № 7, pp.1045-1056.

[9] Galina Mehdiyeva, Vagif Ibrahimov, On the research of multi-step methods with constant coefficients. Monograph, Lambert. acad. publ., 2013

[10] Vagif Ibrahimov, Mehriban Imanova, Galina Mehdiyeva, On the construction of the Multistep Methods to Solving the Initial-Value Problem, IAPE'19, Oxford, United Kingdom

[11] Galina Mehdiyeva, Mehriban Imanova, Vagif Ibrahimov On a one method for determing the maximal value of the order of the accuracy for the multistep hybrid method which have applied to solving of the Volterra integro-differential equation of the first order-2018 
[12] Hairier E., Norsett S.P., Wanner G. Solving ordinary differential equations. (Russian) M., Mir, 1990.

[13] R.B.Ogunrinde, J.O. Olubunmi, Solution of Some Second Order Ordinary Differential Equatons Using a Derived Algorithm

[14] Gelfond, A.O.,Calculus of Finite Differences, 3th edition, "Nauka», Moscow, 1967, pp. 311-319

[15] ButcherJ.C. A modified multistep method for the numerical integration of ordinary differential equations. $J$. Assoc. Comput. Math., v.12, 1965, pp. 124-135.

[16] Gear C.S.,Hybrid methods for initial value problems in ordinary differential equations, Algorithm IS, J. Number. Anal. v. 2, 1965, pp. 69-86.

[17] Dahlquist G., Convergence and stability in the numerical integration of ordinary differential equations, Math. Scand, 1956, No.4, pp. 33-53.

[18] Dahlquist G., Stability and error bounds in the numerical integration of ordinary differential equation.,Trans. Of the Royal Inst. Of Techn., Stockholm, Sweden, Nr. 130, 1959, pp. 3-87.

[19] DA Juraev-On the Cauchy problem for matrix factorizations of the Helmholtz equation

[20] IbrahimovV.R. Convergence of the predictor-corrector methods, Godishnik na Vischite uchebzaved. Applied Mathematics, Sofia Boolgaria (1984), pages 187-197

\section{Creative Commons Attribution License 4.0 (Attribution 4.0 International, CC BY 4.0)}

This article is published under the terms of the Creative Commons Attribution License 4.0

https://creativecommons.org/licenses/by/4.0/deed.en_US 